POS PROCEEDINGS

\title{
Two Aspects of M-(brane) theory
}

\section{Jens Hoppe*}

Sogang University

E-mail: hoppedsogang.ac.kr

The Reconstruction Algebra of [3] is quantized, and a novel approach to Quantum M-branes presented.

Corfu Summer Institute on Elementary Particles and Physics - Workshop on Non Commutative Field Theory and Gravity,

September 8-12, 2010

Corfu Greece

${ }^{*}$ Speaker. 
The attempt to quantize Relativistic M(em)branes (M-dimensional extended objects in Ddimensional space-time) is intimately related to non-commutative field theory and gravity. The fuzzy sphere was invented in this context (cp. [1]), and the hope of including gravity is reflected from many points of view (e.g. [2]). Here I would like to report on 2 topics relevant to this endavour, namely

\section{A) Quantum Reconstruction Algebras}

In a recent paper [3] it was found that the reconstruction [4] of the coordinate which disappears in the light cone description of relativistic extended objects,

$$
\begin{gathered}
\zeta(\varphi)=\zeta_{0}+\frac{1}{\eta} \int G(\varphi, \widetilde{\varphi}) \widetilde{\nabla}^{a}\left(\frac{\vec{p}}{\rho} \widetilde{\nabla}_{a} \vec{x}\right)(\widetilde{\varphi}) \rho(\widetilde{\varphi}) d^{M} \widetilde{\varphi} \\
\int G(\varphi, \widetilde{\varphi}) \rho(\varphi) d^{M} \varphi=0, \widetilde{\triangle} G(\varphi, \widetilde{\varphi})=\frac{\delta(\varphi, \widetilde{\varphi})}{\rho}-1,
\end{gathered}
$$

leads to higher-dimensional generalisations of the Witt-Virasoro algebra, when considering the (classical or quantum) commutation relations of the field $\zeta$ at different points $\varphi$ of the parameter manifold $\Sigma_{M}$ (modulo volume-preserving diffeomorphisms);

Namely

$$
\left[L_{\alpha}, L_{\alpha^{\prime}}\right]=e_{\left[\alpha, \alpha^{\prime}\right] \varepsilon} L_{\varepsilon}
$$

where

$$
e_{\alpha \beta \gamma}:=\frac{\mu_{\beta}-\mu_{\gamma}}{\mu_{\alpha}} \int_{\Sigma_{M}} Y_{\alpha} Y_{\beta} Y_{\gamma} \rho d^{M} \varphi=: \frac{\mu_{\beta}-\mu_{\gamma}}{\mu_{\alpha}} d_{\alpha \beta \gamma}
$$

with $Y_{\alpha}$, resp. $-\mu_{\alpha}(\alpha=1,2, \ldots)$, being the (non-constant) eigenfunctions, resp (negative) eigenvalues of the Laplacian on $\Sigma_{M}, \triangle=\frac{1}{\rho} \partial_{a} \rho h^{a b} \partial_{b}, \sqrt{\operatorname{det} h^{a b}}=\rho$.

In [3] it was shown that (modulo volume-preserving diffeomorphisms)

$$
\eta \zeta_{\alpha}:=\eta \int Y_{\alpha} \zeta(\varphi) \rho d^{M} \varphi, \quad \alpha=1,2, \ldots
$$

form a representation of (2), with [, ] being the classical Poisson bracket. Rewriting (1) as

$$
\begin{gathered}
\zeta=\zeta_{0}+\frac{1}{2 \eta}\left(\frac{\vec{p}}{\rho} \vec{x}-\int \vec{p} \vec{x}\right)+\frac{1}{2 \eta} \int G(\varphi, \widetilde{\varphi})\left(\frac{\vec{p}}{\rho} \Delta \vec{x}-\vec{x} \triangle \frac{\vec{p}}{\rho}\right)(\widetilde{\varphi}) \rho(\widetilde{\varphi}) d^{M} \varphi \\
\widetilde{\zeta}_{\alpha}:=2 \eta \zeta_{\alpha}-2 \vec{P} \vec{x}_{\alpha}
\end{gathered}
$$

one finds that

$$
\left[\widetilde{\zeta}_{\alpha}, \widetilde{\zeta}_{\alpha^{\prime}}\right]=2 e_{[\alpha, \alpha] \varepsilon} \widetilde{\zeta}_{\varepsilon}=\left(e_{\alpha \alpha^{\prime} \varepsilon}-e_{\alpha^{\prime} \alpha \varepsilon}\right) \widetilde{\zeta}_{\varepsilon}
$$


and that $\widetilde{\zeta}_{\alpha}$ can be written as

$$
\widetilde{\zeta}_{\alpha}=\widetilde{D}_{\alpha}+\widetilde{E}_{\alpha}:=\left(d_{\alpha \beta \gamma}+e_{\alpha \beta \gamma}\right) \vec{x}_{\beta} \vec{p}_{\gamma}
$$

Promoting the classical variables, $x_{j \beta}$ and $p_{k \gamma}$, to quantum operators satisfying

$$
\left[x_{j \beta}, p_{k \gamma}\right]=i \delta_{j k} \delta_{\beta \gamma}
$$

it is not difficult to see that

$$
\hat{\zeta}_{\alpha}:=\frac{i}{2}\left(D_{\alpha}+E_{\alpha}\right), 2 D_{\alpha}=d_{\alpha \beta \gamma}\left(\overrightarrow{x_{\beta}} \vec{p}_{\gamma}+\vec{p}_{\gamma} \vec{x}_{\beta}\right), E_{\alpha}=e_{\alpha \beta_{\gamma}} \vec{x}_{\beta} \vec{p}_{\gamma}
$$

will form a representation of (7), with $[$,$] the ordinary commutator of operators. Rather than lifting$ the derivation given in [3] to one involving quantummechanical operators one can also formally verify (7) by showing that

$$
\begin{gathered}
{\left[D_{\alpha}, D_{\alpha^{\prime}}\right]=i\left(\vec{x}_{\alpha} \vec{p}_{\alpha^{\prime}}-\vec{x}_{\alpha^{\prime}} \vec{p}_{\alpha}\right)} \\
{\left[D_{\alpha}, E_{\alpha^{\prime}}\right]+\left[E_{\alpha}, D_{\alpha^{\prime}}\right]=2 i e_{\left[\alpha, \alpha^{\prime}\right] \varepsilon} D_{\varepsilon}} \\
{\left[E_{\alpha}, E_{\alpha^{\prime}}\right] \approx 2 i e_{\left[\alpha, \alpha^{\prime}\right] \varepsilon} E_{\varepsilon}-i\left(\vec{x}_{\alpha} \vec{p}_{\alpha^{\prime}}-\vec{x}_{\alpha^{\prime}} \vec{p}_{\alpha}\right) .}
\end{gathered}
$$

To obtain (11) one simply notes that

$$
d_{\alpha \varepsilon \gamma} d_{\alpha^{\prime} \beta \varepsilon}-d_{\alpha \beta \varepsilon} d_{\alpha^{\prime} \varepsilon \gamma}=-\delta_{\alpha \gamma} \delta_{\alpha^{\prime} \beta}+\delta_{\alpha \beta} \delta_{\alpha^{\prime} \gamma}
$$

due to the completeness-relation

$$
\sum_{\alpha=1}^{\infty} Y_{\alpha}(\varphi) Y_{\alpha}(\widetilde{\varphi})=\frac{\delta(\varphi, \widetilde{\varphi})}{\rho(\varphi)}-1
$$

To obtain (12) one first obtains

$$
2\left[D_{\alpha}, E_{\alpha^{\prime}}\right]=i\left(d_{\alpha \varepsilon \gamma} e_{\alpha^{\prime} \beta \varepsilon}-d_{\alpha \beta \varepsilon} e_{\alpha^{\prime} \varepsilon \gamma}\right)\left(\vec{x}_{\beta} \vec{p}_{\gamma}+\vec{p}_{\gamma} \vec{x}_{\beta}\right)
$$

and then proves that

$$
\begin{aligned}
& e_{\alpha \varepsilon \beta} d_{\alpha^{\prime} \varepsilon \gamma}+e_{\alpha \varepsilon \gamma} d_{\alpha^{\prime} \varepsilon \beta}-e_{\alpha^{\prime} \varepsilon \beta} d_{\alpha \varepsilon \gamma}-e_{\alpha^{\prime} \varepsilon \gamma} d_{\alpha \varepsilon \beta} \\
& =\left(e_{\alpha \alpha^{\prime} \varepsilon}-e_{\alpha^{\prime} \alpha \varepsilon}\right) d_{\varepsilon \beta \gamma},
\end{aligned}
$$

using

$$
e_{\alpha \beta \gamma}=\frac{1}{\mu_{\alpha}} \int Y_{\alpha}\left(Y_{\beta} \triangle Y_{\gamma}-Y_{\gamma} \triangle Y_{\beta}\right) \rho d^{M} \varphi
$$

and (15).

The calculation leading to (2) in [3] then implies that ( modulo volume-preserving diffeomorphisms/topological terms)

$$
e_{\alpha \varepsilon \beta} e_{\alpha^{\prime} \varepsilon \gamma}-e_{\alpha^{\prime} \varepsilon \beta} e_{\alpha \varepsilon \gamma}+\delta_{\alpha \beta} \delta_{\alpha^{\prime} \gamma}-\delta_{\alpha \gamma} \delta_{\alpha^{\prime} \beta} \approx\left(e_{\alpha \alpha^{\prime} \varepsilon}-e_{\alpha^{\prime} \alpha \varepsilon}\right) e_{\varepsilon \beta \gamma}
$$


which proves (13).

For $M=2$, an identity related to (18) has been derived in [5], using a completeness relation that allows to write problematic terms involving

$$
\sum_{\varepsilon} \frac{1}{\mu_{\varepsilon}} \partial_{a} Y_{\varepsilon}(\varphi) \widetilde{\partial}_{b} Y_{\varepsilon}(\widetilde{\varphi})
$$

(appearing also on the r.h.s. of (18)) in terms of harmonic vectorfields, and $\varepsilon_{a a^{\prime}} \partial^{a^{\prime}} Y_{\varepsilon} \varepsilon_{b b^{\prime}} \partial^{b^{\prime}} Y_{\varepsilon}$ (leading to terms proportional to the areapreserving diffeomorphism constraints), and $\delta(\varphi, \widetilde{\varphi})$. Otherwise, the 'trick' is again to use (17) and (15), resp. to write

$$
\begin{aligned}
& \left(e_{\alpha \varepsilon \beta} e_{\alpha^{\prime} \varepsilon \gamma}-e_{\alpha^{\prime} \varepsilon \beta} e_{\alpha \varepsilon \gamma}\right) \mu_{\alpha} \mu_{\alpha^{\prime}} \\
& =\left(\mu_{\varepsilon}^{2}+\mu_{\beta} \mu_{\gamma}-\mu_{\varepsilon}\left(\mu_{\beta}+\mu_{\gamma}\right)\right) d_{\alpha \varepsilon \beta} d_{\alpha^{\prime} \varepsilon \gamma}-\left(\alpha \leftrightarrow \alpha^{\prime}\right) \\
& =\int Y_{\alpha} \triangle Y_{\varepsilon} Y_{\beta} \int Y_{\alpha^{\prime}} \triangle Y_{\varepsilon} Y_{\gamma}+\int Y_{\alpha} Y_{\varepsilon} \triangle Y_{\beta} \int Y_{\alpha^{\prime}} Y_{\varepsilon} \triangle Y_{\gamma} \\
& -\int Y_{\alpha} \triangle Y_{\varepsilon} Y_{\beta} \int Y_{\alpha^{\prime}} Y_{\varepsilon} \triangle Y_{\gamma}-\int Y_{\alpha} Y_{\varepsilon} \triangle Y_{\beta} \int Y_{\alpha^{\prime}} \triangle Y_{\varepsilon} Y_{\gamma}-\left(\alpha \leftrightarrow \alpha^{\prime}\right)
\end{aligned}
$$

and use (15), after integrating by parts in order to have no derivative acting on $Y_{\varepsilon}$. Note that $\int\left(\nabla Y_{\alpha} \nabla Y_{\beta}\right)\left(\nabla Y_{\alpha^{\prime}} \nabla Y_{\gamma}\right)-\left(\alpha \leftrightarrow \alpha^{\prime}\right)$ is equal to $\int\left\{Y_{\alpha}, Y_{\alpha^{\prime}}\right\}\left\{Y_{\beta}, Y_{\gamma}\right\}$ when $M=2$, which has also been observed in [5] (and probably in [6] as well).

B) Codimension 2 Quantum M-branes:

M-branes are known to have special descriptions and properties when the world volume swept out has codimension 1 (cp. [7-12]). Here I would like to propose a route to quantizing M-branes when the codimension is 2 .

The internal (Mass) $)^{2}$ of membranes in D-dimensional Minkowski-space is known $[1,13]$ to be, in orthonormal light-cone gauge, equal to

$$
\mathbb{M}^{2}=\sum_{i=1}^{D-2} \sum_{\alpha=1}^{\infty} p_{i \alpha} p_{i \alpha}+\frac{1}{2} g_{\alpha \beta \gamma} g_{\alpha \beta^{\prime} \gamma^{\prime}} \vec{x}_{\beta} \cdot \vec{x}_{\beta^{\prime}} \vec{x}_{\gamma} \cdot \vec{x}_{\gamma^{\prime}}
$$

where

$$
g_{\alpha \beta \gamma}:=\int Y_{\alpha}\left(\frac{\partial Y_{\beta}}{\partial \varphi^{1}} \frac{\partial Y_{\gamma}}{\partial \varphi^{2}}-\frac{\partial Y_{\beta}}{\partial \varphi^{2}} \frac{\partial Y_{\gamma}}{\partial \varphi^{1}}\right) d^{2} \varphi
$$

are totally antisymmetric structure constants of the Lie-algebra of "area-preserving" (i.e. unit Jacobian) diffeomorphisms. When $D=5$, (21) can be written as

$$
\mathbb{M}^{2}=\sum_{\alpha=1}^{\infty} \vec{a}_{\alpha}^{\dagger} \vec{a}_{\alpha}
$$

where

$$
a_{j \alpha}=i p_{j \alpha}+\frac{1}{2} g_{\alpha \beta \gamma} \varepsilon_{j k l} x_{k \beta} x_{l \gamma}
$$

Motivated in parts by some classical structures observed in [7], Moncrief [8] (though for the codimension one case) argued that (22) may be a good take-off for quantization. 
Note that, at least formally,

$$
\left[\hat{\zeta}_{\alpha}, a_{j \beta}\right]=i\left(d_{\alpha \beta \gamma}+e_{\alpha \beta \gamma}\right) a_{j \gamma}
$$

holds, and that

$$
\Psi_{0}(x):=e^{-\frac{1}{3} \varepsilon_{j k l} g_{\alpha \beta \gamma} x_{j \alpha} x_{k \beta} x_{l \gamma}}
$$

is (formally) annihilated by the quantization of (23) as well as $\hat{\zeta}_{\alpha}$. While the exponent in (25) for the corresponding Matrix-theory is conventionally considered to take all real values - as pointed out by V. Moncrief (years ago, in a discussion at the Albert Einstein Institute) - it is, with the geometric interpretation of enclosed volume at hand, in the continuum theory extremely natural [8] to restrict to strictly negative exponents by choosing a definite orientation.

Leaving for the moment unanswered the very interesting question whether (25) (resp. its supersymmetric analogue) may actually be Lorentz-invariant, in particular annihilated by the crucial mixed generator $\mathbb{M}_{k-}$ (cp. [14]), let me note that by diagonalizing the real-symmetric matrix $S\left(=R \Lambda R^{T}\right)$ appearing in

one has

$$
\begin{gathered}
{\left[a_{j \alpha}, a_{j^{\prime} \alpha^{\prime}}^{\dagger}\right]=2 \varepsilon_{j j^{\prime} k} g_{\alpha \alpha^{\prime} \gamma} x_{k \gamma}=: 2 S_{j \alpha, j^{\prime} \alpha^{\prime}},} \\
a_{J}=\partial_{J}+\frac{1}{2} S_{J L} x_{L}
\end{gathered}
$$

For $M=1$ (string in 4 Dimensions), $S$ (hence $R$ ) are independent of $x$, so that

$$
\begin{array}{r}
A_{K}:=R_{K J}^{T} a_{J}=R_{K J}^{T} \partial_{J}+\frac{1}{2} \\
A_{J}=\tilde{\partial}_{J}+\lambda_{J} \tilde{x}_{J},
\end{array}
$$

and $J$ can be taken as $(j, n)$ while $j=1,2, n \in \mathbb{Z}-\{0\}$. The explicit formulae for $D=4(M=1)$ are:

with

$$
\begin{gathered}
a_{j}=i p_{j}+\varepsilon_{j k} x^{\prime} \\
\hat{a}_{j \alpha}:=\int Y_{\alpha}(\varphi) \hat{a}_{j}=\partial_{j \alpha}+\varepsilon_{j k} r_{\alpha \beta} x_{k \beta}
\end{gathered}
$$

$$
\begin{gathered}
r_{\alpha \beta}=\int_{0}^{2 \pi} Y_{\alpha} Y_{\beta}^{\prime} d \varphi \\
{\left[a_{j \alpha}, a_{j^{\prime} \alpha^{\prime}}^{\dagger}\right]=2 \varepsilon_{j j^{\prime}} r_{\alpha \alpha^{\prime}}=: S_{j \alpha, j^{\prime} \alpha^{\prime}}} \\
\mathbb{M}^{2}=a_{J}^{\dagger} a_{J}
\end{gathered}
$$

and $a_{j \alpha} \Psi_{0}=0$ would give

$$
\Psi_{0} \sim e^{-\frac{1}{2} \varepsilon_{j j^{\prime}} r_{\alpha \alpha^{\prime}} x_{j \alpha} x_{j^{\prime} \alpha^{\prime}}}
$$

where the exponent, $-\frac{1}{2} x_{J} S_{J J^{\prime}} x_{J^{\prime}}=-\frac{1}{2} \tilde{x}_{J} \lambda_{J} \tilde{x}_{J}$, is proportional to the area enclosed by the curve.

\section{Acknowledgements}

I would like to thank J. Fröhlich, F. Laloe, V. Moncrief, S. Theisen and M. Trzetrzelewski for discussions and correspondence and the organizers of Corfu Summer Institute on Elementary Particles and Physics - Workshop on Non Commutative Field Theory and Gravity for their kind invitation. 


\section{References}

[1] J.Hoppe Quantum theory of a massless relativistic surface and a two-dimensional bound state problem PhD Thesis MIT 1982 (http://dspace.mit.edu/handle/1721.1/15717)

[2] E. Witten; Nucl.Phys. B 460 (1996) 335.

T. Banks, W. Fischler, S. Shenker, L. Susskind; Phys.Rev. D 55 (1997) 5112.

[3] J. Hoppe; Phys.Lett.B 695 (2011) 384, doi:10.1016/j.physletb.2010.11.038.

[4] J. Goldstone, unpublished notes.

[5] K. Ezawa, Y. Matsuo, K. Murakami; Prog.Theor.Phys. 98 (1997) 485

[6] S.Melosch, Diplomarbeit, Hamburg University 1990.

[7] J.Hoppe; Canonical 3+1 Description of Relativistic Membranes arXiv:hep-th/9407103

[8] V. Moncrief; Gen. Rel. Grav. (2006) 38(4):561

[9] M. Bordemann, J. Hoppe; Phys. Lett. B317 (1993) 315

[10] M. Bordemann, J. Hoppe; Phys. Lett. B325 (1994) 359

[11] M.Bordemann, J.Hoppe; J.Math.Phys. 39 (1998) 683

[12] J.Hoppe, T.Ratiu; Class.Quant.Grav.14:L45,1997

[13] J.Goldstone, unpublished

[14] J.Hoppe; M-brane dynamical symmetry and quantization arXiv:1101.4334 